University of Wollongong

Research Online

Faculty of Engineering - Papers (Archive)

Faculty of Engineering and Information

Sciences

$15-4-2006$

\title{
Three-dimensional hysteresis of soft magnetic composite
}

Z. W. Lin

University of Technology, Sydney

J. G. Zhu

University of Technology, Sydney

Y. G. Guo

University of Technology, Sydney

Xiaolin Wang

University of Wollongong, xiaolin@uow.edu.au

S. Y. Ding

Nanjing University

Follow this and additional works at: https://ro.uow.edu.au/engpapers

Part of the Engineering Commons

https://ro.uow.edu.au/engpapers/158

\section{Recommended Citation}

Lin, Z. W.; Zhu, J. G.; Guo, Y. G.; Wang, Xiaolin; and Ding, S. Y.: Three-dimensional hysteresis of soft magnetic composite 2006.

https://ro.uow.edu.au/engpapers/158

Research Online is the open access institutional repository for the University of Wollongong. For further information contact the UOW Library: research-pubs@uow.edu.au 


\title{
Three-dimensional hysteresis of soft magnetic composite
}

\author{
Z. W. Lin, ${ }^{\text {a) }}$ J. G. Zhu, and Y. G. Guo \\ Faculty of Engineering, University of Technology, Sydney, P.O. Box 123 Broadway, \\ New South Wales 2007, Australia
}

\author{
X. L. Wang \\ Spintronic and Electronic Materials Group, Institute for Superconducting and Electronic Materials, \\ University of Wollongong, Wollongong, New South Wales 2522, Australia
}

\section{S. Y. Ding}

Department of Physics, Nanjing University, Nanjing 210093, People's Republic of China

(Presented on 31 October 2005; published online 26 April 2006)

\begin{abstract}
Hysteresis phenomenon is one of the important characteristics of soft magnetic materials. A three-dimensional (3D) magnetic property measurement system has been developed. This system can effectively control the locus of the time-varying magnetic flux density vector $\mathbf{B}$ and measure the corresponding magnetic field strength vector $\mathbf{H}$ in a cubic sample of soft magnetic material. The sample involved in this study is a soft magnetic composite sample made of highly pure iron powder. This paper investigates the $\mathbf{B}$ and $\mathbf{H}$ loci in $3 \mathrm{D}$ space and the power loss when the $\mathbf{B}$ loci are controlled to become circles and ellipses lying in three orthogonal planes. It is found that the $\mathbf{B}$ and $\mathbf{H}$ loci lie in the same magnetization plane, but the $\mathbf{H}$ loci and losses depend strongly on the orientation, position, and process of magnetization. On the other hand, the $\mathbf{H}$ vectors evolve into a unique locus pattern, and the power losses approach a unique value when the $\mathbf{B}$ vectors evolve into the same loci through either a series of small circles or ellipses. (C) 2006 American Institute of Physics. [DOI: 10.1063/1.2173947]
\end{abstract}

\section{INTRODUCTION}

Applications of magnetic materials largely rely on the hysteresis characteristics. The hysteresis curves of a material, as a valuable means connecting the magnetization mechanisms and applications of the material, are determined by a complex interplay of the magnetic domain wall motion and the rotation of local magnetization vectors. Studying the curves is of great benefit to build general theories to interpret the hysteresis phenomenon and also to develop practical models for optimum design of innovative electromagnetic devices, for example, electric machines. However, there are very few experiments investigating three-dimensional (3D) characteristics of soft magnetic materials, though great efforts have been made to study one- and two-dimensional magnetic properties. ${ }^{1-4}$ This paper studies 3D experimental results of time-varying magnetic flux density vector B loci and magnetic field strength vector $\mathbf{H}$ loci, and the power losses when the B loci are controlled to become circles with increasing amplitudes and ellipses evolving from a straight line into a circle in three orthogonal planes, respectively.

\section{EXPERIMENTAL SETUP}

A 3D magnetic property measurement system has been developed. ${ }^{5,6}$ This system can effectively control the $\mathbf{B}$ locus and measure the corresponding $\mathbf{H}$ locus in a cubic sample of soft magnetic materials. The system consists of a 3D orthogonal yoke system, a control system, a data acquisition

\footnotetext{
a) Author to whom correspondence should be addressed; electronic mail:
} jacklin@eng.uts.edy.au system, and particular sensors for $\mathbf{B}$ and $\mathbf{H}$ vectors. Six thin $H$ sensors with $0.5 \mathrm{~mm}$ thickness were immediately attached to the sample surfaces to measure the tangential component of the $\mathbf{H}$ field and the normal component of the $\mathbf{B}$ field. The sample with dimensions of $22 \times 22 \times 22 \mathrm{~mm}^{3}$ is a soft magnetic composite material made of highly pure iron powder with surface coating. It has high resistivity to ensure low eddy current loss. In particular, the sample was enclosed by six guard pieces cut from a sample material in order to make the magnetic field inside the sample uniform.

\section{RESULTS AND DISCUSSION}

The alternating hysteresis properties of the sample were studied firstly, and then 3D properties, that is, $\mathbf{B}$ vectors with circular and elliptical loci controlled to lie in the three orthogonal planes and the corresponding $\mathbf{H}$ vectors, were investigated. Figure 1 plots the hysteresis loops with the $\mathbf{B}$

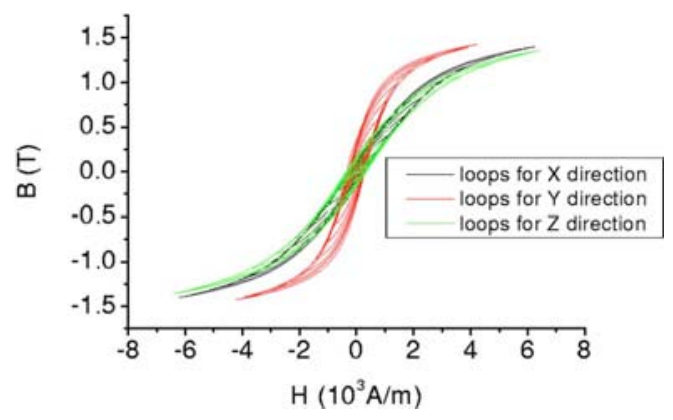

FIG. 1. Alternating hysteresis loops when the sample was magnetized along the $x, y$, and $z$ axes. 


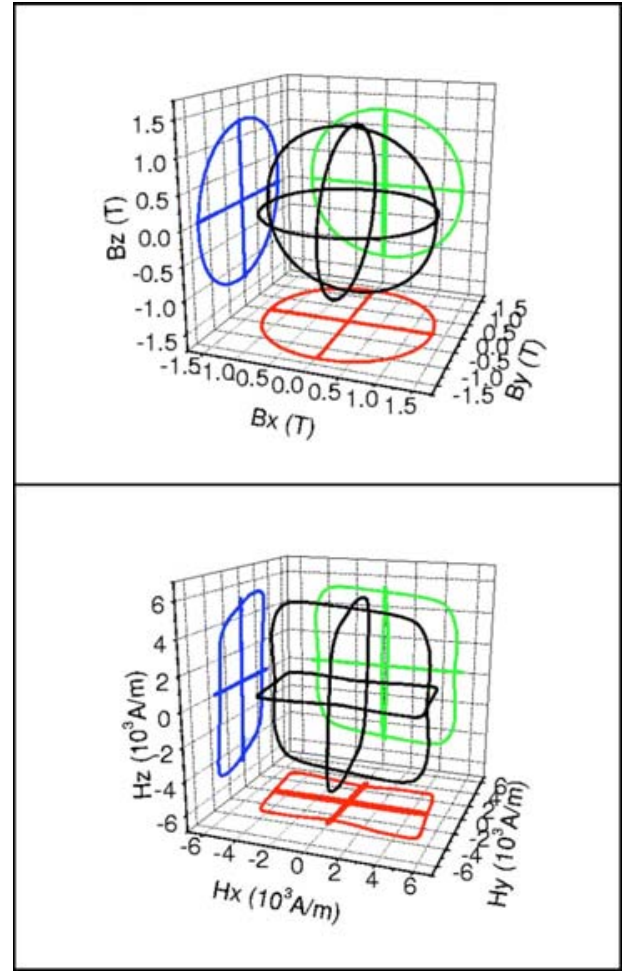

FIG. 2. B (top) and $\mathbf{H}$ (bottom) loci when amplitude of circular $\mathbf{B}$ reaches $1.3 \mathrm{~T}$ in three orthogonal planes. magnitude up to $1.3 \mathrm{~T}$ when the sample was magnetized at $50 \mathrm{~Hz}$ along the $x, y$, and $z$ axes. It is noticed that the loops for the $x$ and $z$ axes are similar, while the $y$ axis seems to be the easy axis, though the sample are expected to be isotropic. The results are in agreement with the data provided by Höganas AB, Sweden.

The controlled circular $\mathbf{B}$ loci and the corresponding $\mathbf{H}$ loci were measured at $50 \mathrm{~Hz}$ when the amplitude of $\mathbf{B}$ increases up to $1.3 \mathrm{~T}$ in the $x o y, y o z$, and zox planes. Figure 2 plots the $\mathbf{B}$ and $\mathbf{H}$ loci when the amplitude of $\mathbf{B}$ reaches 1.3 T. It is found that the $\mathbf{B}$ and $\mathbf{H}$ loci lie in the same magnetization planes. The $\mathbf{H}$ loci in the xoy and yoz planes evolve from ellipses into rectanglelike loops while the $\mathbf{H}$ loci in the zox plane changes from circles into squarelike loops. This phenomenon is consistent with the alternating hysteresis loops since the easy magnetization is along the $y$ axis.

Figure 3 plots the $\mathbf{B}$ and $\mathbf{H}$ loci at $50 \mathrm{~Hz}$ when the $\mathbf{B}$ loci evolve gradually from a straight line into a circle with an amplitude of $1.3 \mathrm{~T}$ via a series of ellipses in the xoy, yoz, and zox planes. Note that the ultimate $\mathbf{B}$ circles evolve from two directions in each plane. It is found that the $\mathbf{H}$ loci also evolve from two directions, but the ultimate $\mathbf{H}$ loci are similar. In the xoy plane, for example, there are two series of elliptical $\mathbf{B}$ loci, whose major axes are along the $x$ or $y$ axis individually, but the ultimate $\mathbf{H}$ loci with respect to the $\mathbf{B}$ loci with the amplitude of $1.3 \mathrm{~T}$ are similar. In addition, both

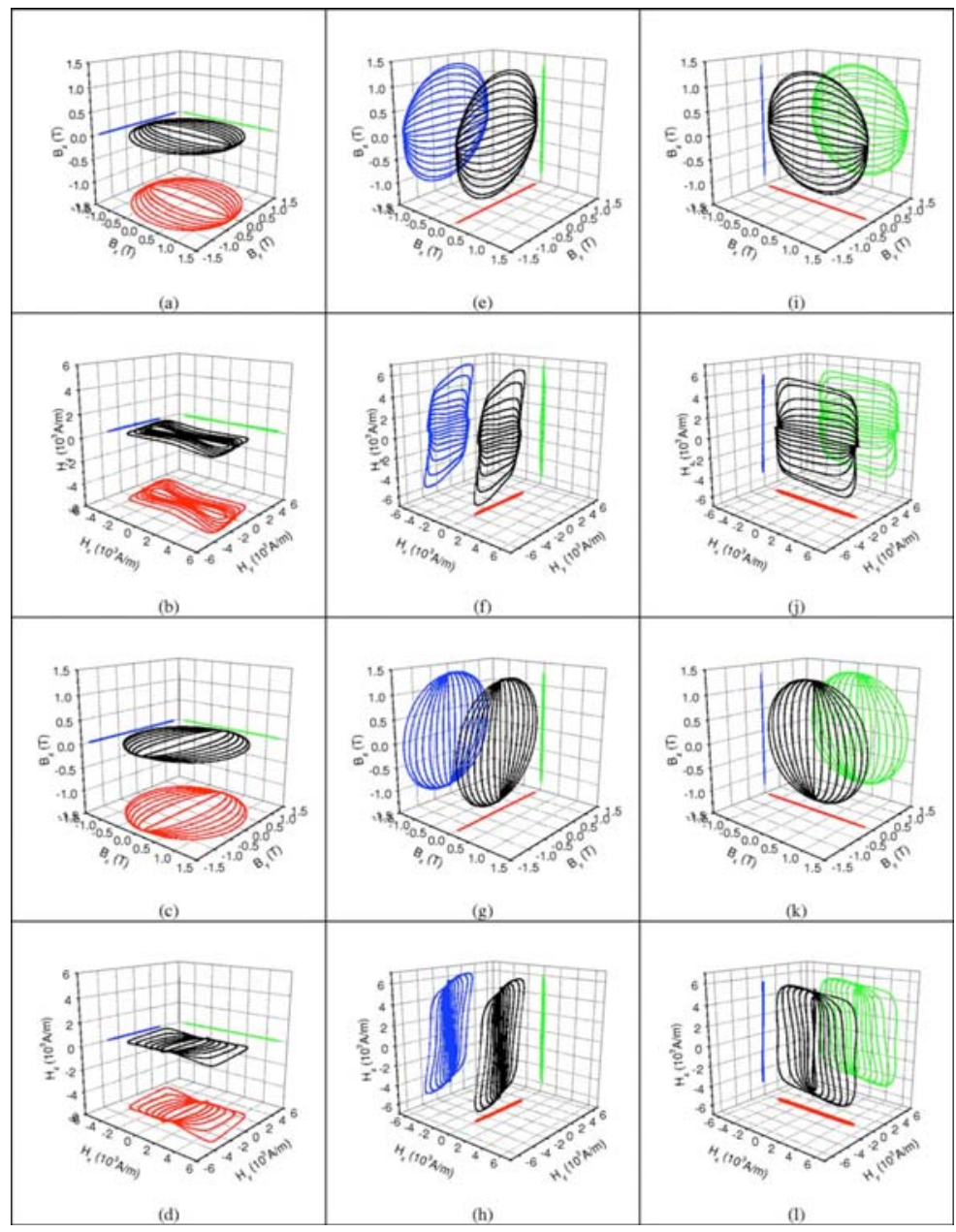

FIG. 3. B (rows 1 and 3) and $\mathbf{H}$ (rows 2 and 4) loci when $\mathbf{B}$ loci evolve from a line to a circle with amplitudes of $1.3 \mathrm{~T}$ in the xoy (left column), yoz (middle column), and zox planes (right column). 


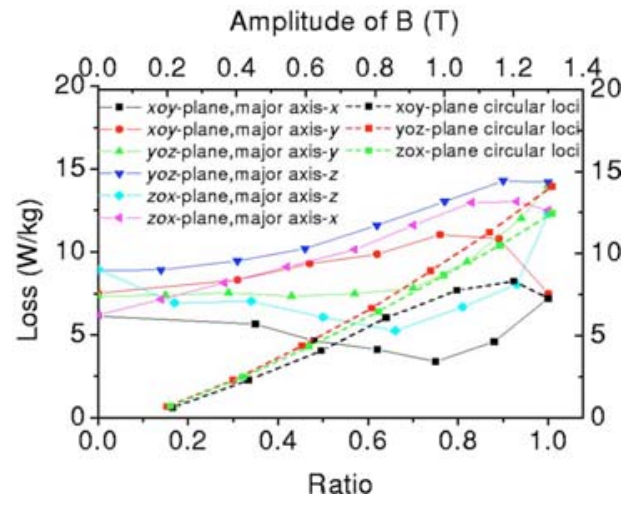

FIG. 4. Power losses vs ratios of minor axis to major axis (bottom axis) of elliptical B loci evolving from lines to circles via ellipses, and vs amplitudes of circular B loci (upper axis)

the ultimate $\mathbf{H}$ loci are similar to the $\mathbf{H}$ locus when the $\mathbf{B}$ locus is circle with the amplitude of $1.3 \mathrm{~T}$, shown in Fig. 2. Similar to Fig. 2, the $\mathbf{H}$ loci, shown in Fig. 3, in the xoy, and yoz planes evolve into rectanglelike loops, while the loci in the zox plane into squarelike loops.

Figure 4 plots the power losses when the B loci evolve from a series of circles and from a straight line into a circle in different planes. The similarity of the ultimate $\mathbf{H}$ loci can also be approved by power losses. For example, the losses approach $7.4 \mathrm{~W} / \mathrm{kg}$ when the $\mathbf{B}$ locus approaches the circle with the amplitude of $1.3 \mathrm{~T}$ in the xoy plane, $14.1 \mathrm{~W} / \mathrm{kg}$ in the yoz plane, and $12.3 \mathrm{~W} / \mathrm{kg}$ in the zox plane. However, the losses depend strongly on the direction of the major axis, the magnetization plane, and the process.

\section{CONCLUSION}

3D magnetic properties of a cubic soft magnetic composite material made of pure iron powder with insulation coating have been studied by measuring the alternating hysteresis loops and 3D spatial $\mathbf{B}$ and $\mathbf{H}$ loci when the $\mathbf{B}$ loci are controlled. It is found that the shape and orientation of the corresponding $\mathbf{H}$ loci and power losses depend strongly on the magnetization plane, direction, and process. However, the ultimate $\mathbf{H}$ vectors have a similar pattern, and the corresponding power losses are similar when the $\mathbf{B}$ vectors evolve into the same loci through either a series of small circles or ellipses.

${ }^{1}$ J. G. Zhu, J. J. Zhong, V. S. Ramsden, and Y. G. Guo, J. Appl. Phys. 85, 4403 (1999).

${ }^{2}$ G. Bertotti, A. Canova, M. Chiampi, D. Chiarabaglio, F. Fiorillo, and A. M. Rietto, J. Magn. Magn. Mater. 133, 647 (1994).

${ }^{3}$ L. R. Dupre, F. Fiorillo, C. Appino, A. M. Rietto, and J. Melkebeek, J. Appl. Phys. 87, 6511 (2000).

${ }^{4}$ J. Sievert, J. Magn. Magn. Mater. 215, 647 (2000).

5 J. G. Zhu, J. J. Zhong, Z. W. Lin, and J. D. Sievert, IEEE Trans. Magn. 39, 3429 (2003).

${ }^{6}$ Z. W. Lin, H. W. Lu, J. G. Zhu, J. J. Zhong, X. L. Wang, and S. Y. Ding, J. Appl. Phys. 97, 10R306-1 (2005). 